Bull. Austral. Math. Soc.

$16 \mathrm{P} 40$

VoL. $62(2000)$ [51-56]

\title{
RELATIVE CONTINUITY OF DIRECT SUMS OF $M$-INJECTIVE MODULES
}

\author{
LiU Zhongkui and Javed Ahsan
}

\begin{abstract}
Let $M$ be a left $R$-module and $\mathcal{K}$ be an $M$-natural class with some additional conditions. It is proved that every direct sum of $M$-injective left $R$-modules in $\mathcal{K}$ is $\mathcal{K S}$-continuous ( $\mathcal{K S}$-quasi-continuous) if and only if every direct sum of $M$ injective left $R$-modules in $\mathcal{K}$ is $M$-injective.
\end{abstract}

Let $R$ be a ring with identity. It is well-known that $R$ is left Noetherian if and only if every direct sum of injective left $R$-modules is injective. Based on this, many characterisations of left Noetherian rings using generalised injectivity of some left $R$ modules have been obtained. For example, it was shown that $R$ is left Noetherian if and only if every direct sum of injective left $R$-modules is continuous (or quasi-continuous) (see [5]). On the other hand, Albu, Nastasescu, Golan, Goldman, Stenstrom, Teply, Enochs, Ahsan and others have studied the situations when all direct sums of nonsingular injective left $R$-modules are injective, when all direct sums of $\tau$-torsion free injective left $R$-modules are injective for a hereditary torsion theory $\tau$, and when all direct sums of $\tau$-torsion injective left $R$-modules are injective for a stable hereditary torsion theory $\tau$. These results are well presented in Golan's book [4], and have been generalised in [12] by considering when all direct sums of $M$-injective left $R$-modules in an $M$-natural class $\mathcal{K}$ are $M$-injective. In this paper we consider when all direct sums of $M$-injective left $R$-modules in an $M$-natural class $\mathcal{K}$ are $\mathcal{K} \mathcal{S}$-continuous (or $\mathcal{K S}$-quasi-continuous). We shall show that for an $M$-natural class $\mathcal{K}$, all direct sums of $M$-injective left $R$-modules in $\mathcal{K}$ are $\mathcal{K} \mathcal{S}$-continuous (or $\mathcal{K} \mathcal{S}$-quasi-continuous) if and only if all direct sums of $M$-injective left $R$-modules in $\mathcal{K}$ are $M$-injective.

Throughout this note we write $A \leq_{e} B(A \mid B)$ to denote that $A$ is an essential submodule (a direct summand) of $B$.

Let $M$ be a left $R$-module. We say that a left $R$-module $N$ is subgenerated by $M$, or that $M$ is a subgenerator for $N$, if $N$ is isomorphic to a submodule of an $M$ generated module. Following [11], we denote by $\sigma[M]$ the full subcategory of $R$-Mod whose objects are all $R$-modules subgenerated by $M$. By $[11,17.9]$, every module $N$

Received 21st September, 1999

The first author was supported by the National Science Foundation of China (19671063).

Copyright Clearance Centre, Inc. Serial-fee code: 0004-9727/00 \$A2.00+0.00. 
in $\sigma[M]$ has an injective hull $I(N)$ in $\sigma[M]$, which is also called an $M$-injective hull of $N$. It is known that the $M$-injective hulls of a left $R$-module in $\sigma[M]$ are unique up to isomorphism. In the following, we always denote by $I(N)$ the $M$-injective hull of $N$ for any left $R$-module $N \in \sigma[M]$.

According to [12], a subclass $\mathcal{K}$ in $\sigma[M]$ which is closed under submodules, direct sums, isomorphic copies, and $M$-injective hulls is called an $M$-natural class. There exist a large number of examples of $M$-natural classes. Among them are $\sigma[M]$ and all natural classes in the sense of [9]. In particular, hereditary torsionfree classes, stable hereditary torsion classes, and saturated classes in the sense of Dauns (see [1]) are examples of $M$-natural classes.

For an $M$-natural class $\mathcal{K}$ and a left $R$-module $N$, we denote by $H_{\mathcal{K}}(N)$ the set $\{L \leq N \mid N / L \in \mathcal{K}\}$.

Let $M, N$ be left $R$-modules. Define the family

$$
\mathcal{A}(N, M)=\left\{A \subseteq M \mid \exists X \subseteq N, \exists f \in \operatorname{Hom}(X, M), f(X) \leq_{e} A\right\} .
$$

Consider the properties

$\mathcal{A}(N, M)-\left(C_{1}\right):$ For all $A \in \mathcal{A}(N, M), \exists A^{*} \mid M$, such that $A \leq_{e} A^{*}$.

$\mathcal{A}(N, M)-\left(C_{2}\right):$ For all $A \in \mathcal{A}(N, M)$, if $X \mid M$ is such that $A \cong X$, then $A \mid M$.

$\mathcal{A}(N, M)-\left(C_{3}\right):$ For all $A \in \mathcal{A}(N, M)$ and $X \mid M$, if $A \mid M$ and $A \cap X=0$ then $A \oplus X \mid M$.

According to [7], $M$ is said to be $N$-extending, $N$-quasi-continuous or $N$ continuous, respectively, if $M$ satisfies $\mathcal{A}(N, M)-\left(C_{1}\right), \mathcal{A}(N, M)-\left(C_{1}\right)$ and $\mathcal{A}(N, M)$ $\left(C_{3}\right), \mathcal{A}(N, M)-\left(C_{1}\right)$ and $\mathcal{A}(N, M)-\left(C_{2}\right)$.

Lemma 1. [7, Proposition 2.4] $A$ left $R$-module $M$ is (quasi-)continuous (see [2]) if and only if $M$ is $M$-(quasi-)continuous if and only if $M$ is $N$-(quasi-)continuous for every left $R$-module $N$.

Given an $M$-natural class $\mathcal{K}$, a left $R$-module $N$ is called $\mathcal{K}$-cocritical if $N \in \mathcal{K}$ and $N / P \notin \mathcal{K}$ for any $0 \neq P \subset N$.

Definition 2: Let $\mathcal{K}$ be an $M$-natural class. A left $R$-module $M$ is said to be $\mathcal{K S}$-extending, $\mathcal{K S}$-quasi-continuous or $\mathcal{K S}$-continuous, respectively, if for any direct sum $C=\bigoplus_{i \in I} C_{i}$ of $\mathcal{K}$-cocritical modules $C_{i}(i \in I), M$ is $C$-extending, $C$-quasicontinuous or $C$-continuous.

Clearly (quasi-)continuous modules are $\mathcal{K S}$-(quasi-)continuous. But the following example shows that the converse is not true.

Example 3. (See [6].) Let $R$ be a left Noetherian V-ring which is not Artinian semisimple (see, for example, [3]). Then, by [7, Corollary 3.7], every left $R$-module 
is $N$-continuous for every semisimple left $R$-module $N$. Thus every left $R$-module is $\mathcal{K S}$-continuous, where $\mathcal{K}=R$-Mod. If all left $R$-modules are quasi-continuous, then for every left $R$-module $M, M \oplus E(M)$ is quasi-continuous, and so $M$ is injective by [8, Lemma $\mathrm{C}]$, where $E(M)$ denotes the injective hull of $M$. Thus $R$ is Artinian semisimple, a contradiction. Hence there exists a left $R$-module $M$ which is not quasicontinuous.

LEMma 4. Any direct summand of a $\mathcal{K S}$-continuous (KS-quasi-continuous) left $R$-module is $\mathcal{K S}$-continuous ( $\mathcal{K S}$-quasi-continuous).

Proof: This follows from the fact that condition $\mathcal{A}(N, M)-\left(C_{i}\right),(i=1,2,3)$ is inherited by direct summands of $M$ [7, Proposition 2.4].

Lemma 5. [7] If $M$ is $N$-(quasi-)continuous and $A \in \mathcal{A}(N, M)$ is a direct summand of $M$ then $A$ is indeed (quasi-)continuous.

Let $c$ be any cardinal. A left $R$-module $M$ is called $c$-limited provided every direct sum of non-zero submodules of $M$ contains at most $c$ direct summands [10].

We say an $M$-natural class $\mathcal{K}$ satisfies $\left({ }^{*}\right)$ (see [12]), if for any cyclic submodule $N$ of $M$, and every ascending chain $N_{1} \leq N_{2} \leq \ldots \ldots$ with each $N_{i} \in H_{\mathcal{K}}(N)$, the union $\bigcup_{i} N_{i}$ belongs to $H_{\mathcal{K}}(N)$.

THEOREM 6. The following conditions are equivalent for an $M$-natural class $\mathcal{K}$ with $\left({ }^{*}\right)$.

(1) $H_{\mathcal{K}}(A)$ has $A C C$ for any cyclic (or finitely generated) submodule $A$ of $M$.

(2) Every direct sum of $M$-injective left $R$-modules in $\mathcal{K}$ is $M$-injective.

(3) Every direct sum of $M$-injective left $R$-modules in $\mathcal{K}$ is $\mathcal{K S}$-continuous.

(4) Every direct sum of $M$-injective left $R$-modules in $\mathcal{K}$ is $\mathcal{K S}$-quasicontinuous.

(5) There exists a cardinal $c$ such that every direct sum of $M$-injective left $R$-modules in $\mathcal{K}$ is the direct sum of a c-limited module and a $\mathcal{K S}$ continuous module.

(6) There exists a cardinal $c$ such that every direct sum of $M$-injective left $R$-modules in $\mathcal{K}$ is the direct sum of a $c$-limited module and a $\mathcal{K S}$-quasicontinuous module.

Proof: (1) $\Longleftrightarrow(2)$. This follows from [12, Theorem 2.4].

(2) $\Longrightarrow(3)$. Suppose that $N=\bigoplus_{i \in I} N_{i}$ is the direct sum of $M$-injective left $R$ modules $N_{i} \in \mathcal{K}, i \in I$. Then $N$ is $M$-injective by (2). On the other hand, $N$ is in $\mathcal{K}$, and so $N \in \sigma[M]$. Thus $N$ is quasi-injective. Now clearly $N$ is $\mathcal{K S}$-continuous by Lemma 1. 
(3) $\Longrightarrow(4)$ is clear.

$(4) \Longrightarrow(1)$. By [12, Theorem 2.5], it is sufficient to show that every direct sum of $M$-injective hulls of $\mathcal{K}$-cocritical left $R$-modules is $M$-injective.

Let $C_{i}, i \in I$, be $\mathcal{K}$-cocritical left $R$-modules. Then $C_{i} \in \mathcal{K}, i \in I$. Set

$$
\begin{gathered}
N=\left(\bigoplus_{i \in I} I\left(C_{i}\right)\right) \oplus I\left(\bigoplus_{i \in I} I\left(C_{i}\right)\right), \\
L=N \oplus I(N) .
\end{gathered}
$$

Then clearly $L$ is a direct sum of $M$-injective left $R$-modules. Since $\mathcal{K}$ is closed under direct sums and $M$-injective hulls, it follows that $L$ is a direct sum of $M$-injective left $R$-modules in $\mathcal{K}$. Thus $L$ is $\mathcal{K S}$-quasi-continuous. Denote

$$
S=\left(\bigoplus_{i \in I} C_{i}\right) \bigoplus\left(\bigoplus_{i \in I} C_{i}\right)
$$

Then $L$ is $S$-quasi-continuous. For the submodule $A=N \bigoplus 0$ of $L$, define an $R$ homomorphism $f: S \longrightarrow L$ as the induced $R$-homomorphism

$$
S=\left(\bigoplus_{i \in I} C_{i}\right) \bigoplus\left(\bigoplus_{i \in I} C_{i}\right) \rightarrow\left(\bigoplus_{i \in I} I\left(C_{i}\right)\right) \oplus I\left(\bigoplus_{i \in I} I\left(C_{i}\right)\right) \oplus 0
$$

(by the natural maps $C_{i} \longrightarrow I\left(C_{i}\right)$ and $\bigoplus_{i \in I} C_{i} \longrightarrow I\left(\bigoplus_{i \in I} I\left(C_{i}\right)\right)$ ). Since $C_{i} \leq_{e} I\left(C_{i}\right)$, we have

$$
\bigoplus_{i \in I} C_{i} \leq_{e} \bigoplus_{i \in I} I\left(C_{i}\right) \leq_{e} I\left(\bigoplus_{i \in I} I\left(C_{i}\right)\right)
$$

Thus

$$
\begin{aligned}
f(S) & =\left(\left(\bigoplus_{i \in I} C_{i}\right) \oplus\left(\bigoplus_{i \in I} C_{i}\right)\right) \oplus 0 \\
& \leq e\left(\left(\bigoplus_{i \in I} I\left(C_{i}\right)\right) \oplus I\left(\bigoplus_{i \in I} I\left(C_{i}\right)\right)\right) \oplus^{0}=A
\end{aligned}
$$

This means that $A \in \mathcal{A}(S, L)$. By Lemma 5 , it follows that $A$ is quasi-continuous. Thus $N$ is quasi-continuous. By $[8$, Lemma $C], \bigoplus_{i \in I} I\left(C_{i}\right)$ is $I\left(\bigoplus_{i \in I} I\left(C_{i}\right)\right)$-injective. Hence $\bigoplus_{i \in I} I\left(C_{i}\right)$ is $M$-injective.

The implications $(3) \Longrightarrow(5) \Longrightarrow(6)$ are clear. 
$(6) \Longrightarrow(4)$. Note that, by Lemma 4 , any direct summand of a $\mathcal{K S}$-quasi-continuous left $R$-module is $\mathcal{K S}$-quasi-continuous. By analogy with the proof of [12, Theorem 2.6], we can complete the proof.

We denote by $\mathcal{S}^{2}$ the class of all semisimple left $R$-modules in $\sigma[M]$.

COROLlaRY 7. The following conditions are equivalent for a left $R$-module $M$.

(1) $M$ is a locally Noetherian module (that is, every finitely generated submodule of $M$ is Noetherian).

(2) Every direct sum of $M$-injective left $R$-modules in $\sigma[M]$ is $M$-injective.

(3) Every direct sum of $M$-injective left $R$-modules in $\sigma[M]$ is $\mathcal{S}^{2}$-continuous.

(4) Every direct sum of $M$-injective left $R$-modules in $\sigma[M]$ is $\mathcal{S}^{2}$-quasicontinuous.

(5) There exists a cardinal $c$ such that every direct sum of $M$-injective left $R$-modules in $\sigma[M]$ is the direct sum of a $c$-limited module and an $\mathcal{S}^{2}$ continuous module.

(6) There exists a cardinal $c$ such that every direct sum of $M$-injective left $R$-modules in $\sigma[M]$ is the direct sum of a $c$-limited module and an $S^{2}$ quasi-continuous module.

COROLlary 8. Let $\mathcal{S}^{2}$ be the class of all semisimple left $R$-modules. The following conditions are equivalent.

(1) $R$ is a left Noetherian ring.

(2) Every direct sum of injective left $R$-modules is $\mathcal{S}^{2}$-continuous $\left(\mathcal{S}^{2}\right.$-quasicontinuous).

(3) There exists a cardinal $c$ such that every direct sum of injective left $R$ modules is the direct sum of a $c$-limited module and an $\mathcal{S}^{2}$-continuous $\left(\mathcal{S}^{2}\right.$-quasi-continuous) module.

Given a stable hereditary torsion theory $\tau$ on $R$-Mod, many equivalent conditions were presented in [9] and [12] to characterise rings which have ACC on $\tau$-dense left ideals. Here we have

Corollary 9. Let $\tau$ be a stable hereditary torsion theory on $R$-Mod and $\tau S$ be the class of all $\tau$-torsion semisimple left $R$-modules. Then the following conditions are equivalent.

(1) $R$ has ACC on $\tau$-dense left ideals.

(2) Every direct sum of $\tau$-torsion injective left $R$-modules is injective.

(3) Every direct sum of $\tau$-torsion injective left $R$-modules is $\mathcal{T S}$-continuous. 
(4) Every direct sum of $\tau$-torsion injective left $R$-modules is $\mathcal{T S}$-quasicontinuous.

(5) There exists a cardinal $c$ such that every direct sum of $\tau$-torsion injective left $R$-modules is the direct sum of a $c$-limited module and a $\mathcal{T S}$-continuous module.

(6) There exists a cardinal $c$ such that every direct sum of $\tau$-torsion injective left $R$-modules is the direct sum of a $c$-limited module and a $T \mathcal{S}$-quasicontinuous module.

\section{REFERENCES}

[1] J. Dauns, 'Classes of modules', Forum Math. 3 (1991), 327-338.

[2] N.V. Dung, D.V. Huynh, P.F. Smith and R. Wisbauer, Extending modules, Pitman Research Notes in Math. 313 (Longman Sci. and Tech., Harlow, 1994).

[3] J.H. Cozzens and J.L. Johnson, 'An application of differential algebra to ring theory', Proc. Amer. Math. Soc. 31 (1972), 354-356.

[4] J.S. Golan, Torsion theories, Pitman Monographs and Surveys in Pure and Applied Mathematics 29 (Longman Sci. and Tech., Harlow, 1986).

[5] Z. Liu, 'Characterizations of rings by their modules', Comm. Algebra 21 (1993), 3663-3671.

[6] Z. Liu, 'Characterization of V-modules by relative quasi-continuity', (submitted).

[7] S.R. Lopez-Permouth, K. Oshiro and S. Tariq Rizvi, 'On the relative (quasi-)continuity of modules', Comm. Algebra 26 (1998), 3497-3510.

[8] B.L. Osofsky and P.F. Smith, 'Cyclic modules whose quotients have all complement submodules direct summands', J. Algebra 139 (1991), 342-354.

[9] S.S. Page and Y. Zhou, 'Direct sums of injective modules and chain conditions', Canad. J. Math. 46 (1994), 634-647.

[10] D. Van Huynh and P.F. Smith, 'Some rings characterised by their modules', Comm. Algebra 18 (1990), 1971-1988.

[11] R. Wisbauer, Foundations of module and ring theory, Algebra, Logic and Applications 3 (Gordon and Breach Science Publishers, Philadelphia P.A., 1991).

[12] Y. Zhou, 'Direct sums of $M$-injective modules and module classes', Comm. Algebra 23 (1995), 927-940.

Department of Mathematics

Northwest Normal University

Lanzhou

Gansu 730070

People's Republic of China
Department of Mathematical Sciences

King Fahd University of Petroleum and Minerals

Dhahran 31261

Saudi Arabia 